\title{
Do tropical rain forest soils have greater nematode diversity than High Arctic tundra? A metagenetic comparison of Malaysia and Svalbard
}

\begin{abstract}
Aim

Latitudinal differences in diversity have long fascinated ecologists. It is unclear whether small soil animals such as nematodes show latitudinal differences in diversity. Based on hypotheses concerning the basis for greater tropical diversity, and by analogy with other groups, we predicted (1) greater alpha diversity in the equatorial tropics than the High Arctic, (2) greater beta and gamma diversity in the tropics. We also predicted (3) no species overlap between the tropics and the High Arctic, and (4) lower abundance of root feeding and insect parasitic nematodes in the high Arctic.

\section{Location}

We sampled at three sites in equatorial rain forest in Malaysia at $2^{\circ} \mathrm{N}$ and three sites in High Arctic tundra in Svalbard at $79^{\circ} \mathrm{N}$.

\section{Methods}

In Malaysia and Svalbard, three sets of $1-\mathrm{m}^{2}$ samples were taken, in interrupted grids. Nematodes were extracted using a Baermann funnel. Bulk DNA was amplified by polymerase chain reaction for the $18 \mathrm{~S}$ rRNA gene, followed by 454-pyrosequencing.

\section{Results}

Comparing operational taxonomic unit (OTU)-based nematode communities (99\% sequence similarity), averaged alpha diversity per $1-\mathrm{m}^{2}$ quadrat showed no difference between Malaysia and Svalbard. However, the classic beta diversity and gamma diversity of nematodes was higher for Malaysia.

\section{Main conclusions}

There was, surprisingly, no difference in the alpha diversity of nematodes between Malaysia and Svalbard. However, for beta and gamma diversity, diversity was higher in Malaysia. There was also considerable OTU overlap between the two regions, suggesting that very broad climatic niches are common in nematode ecology. Plant root feeders and insect parasites were relatively more common in Svalbard. Overall, we found a mixed and complex picture in terms of agreement with what would be predicted on the basis of ecological theory and observations on other groups. While further confirmatory studies would be beneficial, there is a need to incorporate these new findings in the formulation of hypotheses and tests for the underlying causes of latitudinal trends.
\end{abstract}

Keyword: Arctic; Diversity; DNA; Latitude; Malaysia; Metagenetics; Nemat odes; Soil; Tropical; Svalbard 